\title{
Steady shear viscosity of semi-dilute bubbly suspensions
}

\author{
Romain Morini ${ }^{\mathrm{a}}$, Xavier Chateau ${ }^{\mathrm{a}}$, Guillaume Ovarlez ${ }^{\mathrm{a}, \mathrm{b}, *}$, Olivier Pitois ${ }^{\mathrm{a}}$, Laurent Tocquer ${ }^{\mathrm{a}}$ \\ ${ }^{a}$ Université Paris Est, Laboratoire Navier, UMR 8205 CNRS - Ecole des Ponts ParisTech - IFSTTAR, 5 bd Descartes, 77454 Marne-la-Vallée Cedex 2, France \\ ${ }^{\mathrm{b}}$ Université de Bordeaux, CNRS, Solvay, LOF, UMR 5258, Pessac F-33600, France
}

\section{A B S T R A C T}

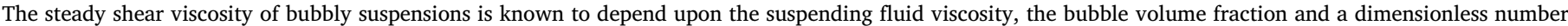

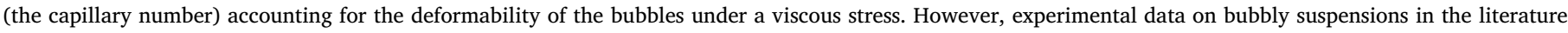

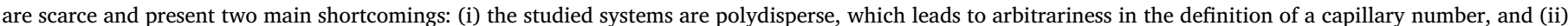

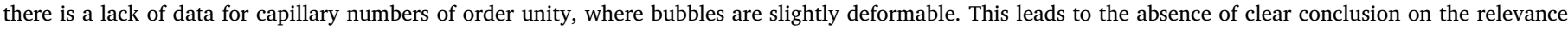

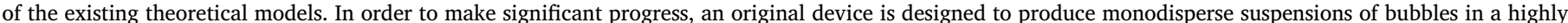

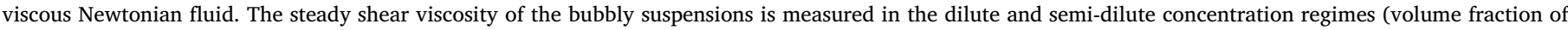

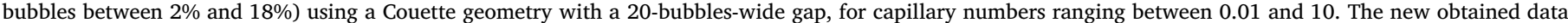

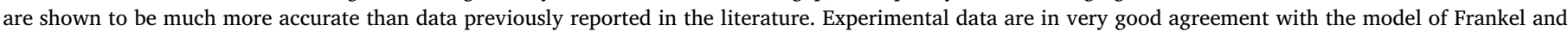
Acrivos (1970), originally developed for dilute suspensions, in the whole range of studied volume fractions.

\section{Introduction}

Understanding the behavior of suspensions of particles, bubbles and droplets, is of high importance for application in the industry (fresh concrete, foodstuff, cosmetic products, plaster foams...) and in the environment (mudslides, lava flows...). A key point is to be able to predict their behavior as a function of their content.

For rigid nonBrownian particles dispersed in a Newtonian fluid, the suspension has a linear behavior but is non-Newtonian (normal stress differences proportional to the shear rate emerge [1]). Its shear viscosity $\eta_{s}(\phi)$ is proportional to the suspending fluid viscosity $\eta_{0}$ and increases with the particle volume fraction $\phi$. In the dilute regime, the dimensionless viscosity is given by the Einstein equation [2]:

$\eta_{s} / \eta_{0}=1+2.5 \phi$

At high volume fractions, many phenomenological expressions exist, among which the Krieger-Dougherty equation [1], which describes the divergence of the viscosity at a given maximum volume fraction $\phi_{m}$, is the most widely used:

$\eta_{s} / \eta_{0}=\left(1-\frac{\phi}{\phi_{m}}\right)^{-2.5 \phi_{m}}$

This equation was built to account for the viscosity divergence while remaining consistent with the Einstein equation at low $\phi$.

For suspensions of bubbles, in addition, the shear viscosity depends on the applied stress. Indeed, in the limit of infinitely low stresses, bubbles behave similarly to rigid particles, with a difference on the boundary condition (no slip at the interface of a rigid particle, slip at the interface of a bubble); the shear viscosity is then found to increase with the bubble volume fraction. In the limit of infinitely high stresses, bubbles are deformed and the viscosity is found to decrease with the bubble volume fraction. At a given volume fraction, the suspension then has a shear-thinning behavior between two viscosity plateaus.

The shear-induced deformation of bubbles is accounted for by a dimensionless number, the capillary number $\mathrm{Ca}$, which compares the viscous stress at the origin of the bubble deformation, and the capillary stress resisting to deformation:

$C a=\tau R / \Gamma$

with $R$ the bubble radius, $\tau$ the shear stress applied to the suspension, and $\Gamma$ the air/liquid surface tension.

For dilute bubble suspensions, the behavior in the limit of nondeformable $(C a \ll 1)$ and fully deformable $(\mathrm{Ca} \gg 1)$ bubbles is theoretically known [3,4]:

$\eta_{s} / \eta_{0}=1+\phi \quad$ for $C a \ll 1$

$\eta_{s} / \eta_{0}=1-5 \phi / 3 \quad$ for $C a \gg 1$

Several models have been proposed to account for the behavior of bubbly suspensions for any capillary number [4-9].

The steady-state response of dilute (i.e., at first order in $\phi$ ) emulsions, of which bubbly suspensions are a special case, has first been computed by Oldroyd [5]. This has later been extended to time-dependent flows by Frankel and Acrivos [4], with rigorous development at first order in the droplet deformation. For bubbly suspensions, the steady-state viscosity

\footnotetext{
* Corresponding author at: Université de Bordeaux, CNRS, Solvay, LOF, UMR 5258, Pessac F-33600, France.

E-mail address: guillaume.ovarlez@u-bordeaux.fr (G. Ovarlez).
} 
in the Frankel and Acrivos (1970) model reads

$\eta_{s} / \eta_{0}=1+\frac{1-(12 / 5) C a^{2}}{1+(6 C a / 5)^{2}} \phi$

Beyond the dilute limit, several attempts have been made to derive a constitutive behavior with the help of homogenization techniques $[6,8,9]$.

Pal [8] has proposed an expansion of the Frankel and Acrivos (1970) model for concentrated suspensions based on the same idea as the Krieger-Dougherty equation. The steady-state viscosity in this model reads

$\eta_{s} / \eta_{0}=\left(1-\frac{\phi}{\phi_{m}}\right)^{-\phi_{m}} \frac{1+K_{1} K_{2}(6 C a / 5)^{2}}{1+K_{1}^{2}(6 C a / 5)^{2}}$

with $K_{1}=\left(1-\frac{\phi}{\phi_{m}}\right)^{-\frac{16}{15} \phi_{m}}, K_{2}=\left(1-\frac{\phi}{\phi_{m}}\right)^{\frac{8}{5}} \phi_{m}$. For monodisperse bubbles, a value of $\phi_{m} \simeq 0.64$ is expected [10].

Faroughi and Huber [9] have used an incremental approach to compute the properties of concentrated suspensions, which leads to an implicit equation for the steady-state viscosity:

$\eta_{s} / \eta_{0}\left(\frac{1-\left(\frac{5}{3}\right)\left(\frac{6 C a}{5}\right)^{2}\left(\eta_{s} / \eta_{0}\right)^{2}}{1-\left(\frac{5}{3}\right)\left(\frac{6 C a}{5}\right)^{2}}\right)^{-\frac{4}{5}}=\left(\frac{\phi_{m}-\phi}{\phi_{m}(1-\phi)}\right)^{-\frac{\phi_{m}}{1-\phi_{m}}}$

also with $\phi_{m} \simeq 0.64$.

All these models are consistent at first order in $\phi$, with in particular a predicted crossover of all curves at a value $\eta_{s} / \eta_{0}=1$ for a critical capillary number independent of $\phi$ :

$C a_{c}=\sqrt{5 / 12} \simeq 0.65$

However, these models provide slightly different predictions in the semi-dilute regime studied here, where the Frankel and Acrivos (1970) model is not expected to be relevant.

There also exists an empirical model developed by Rust and Manga [7] to be consistent with their experimental data:

$\eta_{s} / \eta_{0}=\eta_{s, \infty} / \eta_{0}+\frac{\eta_{s, 0} / \eta_{0}-\eta_{s, \infty} / \eta_{0}}{1+(0.72 C a)^{1.43}}$

with $\eta_{s, \infty} / \eta_{0}=1-1.14 \phi-9.8 \phi^{2}, \eta_{s, 0} / \eta_{0}=\left(1-\frac{\phi}{\phi_{m}}\right)^{-\phi_{m}}$ and $\phi_{m}=0.6$. It should be noted that, in the dilute limit, this model is not consistent with the Frankel and Acrivos (1970) model and predicts a crossover of all curves at a value $\eta_{s} / \eta_{0}=1$ for $C a_{c} \simeq 1.27$.

Model bubble suspensions have been studied experimentally in $[7,11,12,14]$.

Llewellin et al. [11] have studied the unsteady behavior of suspensions of nitrogen bubbles in a golden syrup (with a viscosity of a few tens of Pa.s), obtained with a commercial aerator. The resulting suspension is very polydisperse, with bubble diameter ranging from 2 to $200 \mu \mathrm{m}$. Bubble volume fraction is varied between 0.036 and 0.46 . They have measured the suspension response under oscillations of small strain amplitude, which is ideal for testing the Frankel and Acrivos (1970) model in its strict domain of validity. In these conditions, the behavior is shown to be governed by the dynamic capillary number $\mathrm{Cd}=\frac{\eta_{0} \omega R}{\Gamma}$, where $\omega$ is the angular frequency of the applied oscillations; the dynamic $\eta^{\prime}(\mathrm{Cd})$ response should then be similar to the steady $\eta(\mathrm{Ca})$ response. However, an arbitrary choice is introduced to take into account the large polydispersity of the suspension, through the way viscosity is computed as an average of the contributions of the various bubble diameters (and thus capillary numbers) present in the material; several possible weights depending on the bubble diameter are proposed to compute this average, which makes the comparison to theoretical models somehow unsatisfactory.

Rust and Manga [7] have studied the steady state viscosity of bubbly suspensions in the range of capillary numbers [0.09-3] and have found their results to be well fitted to a phenomenological model (Eq. (10)) they have developed. Their system is made up of a very viscous Newtonian fluid ( $\eta_{0}=180$ Pa.s) in which bubbles are obtained by mixing. This leads to very polydisperse suspensions (the exact polydispersity is not reported, but a picture shows that the diameter varies over more than one order of magnitude, typically between 50 and $500 \mu \mathrm{m}$ ). Several shortcomings can be noted: (i) their results are very scattered for $\mathrm{Ca}<0.3$, (ii) there is a lack of data at high capillary numbers, (iii) the system polydispersity makes the definition of a capillary number tricky and is not taken into account in the comparison to the models by contrast with [11] and [12] ( $\mathrm{Ca}$ is computed with an arbitrary surface-averaged bubble radius), (iv) the stress (and thus capillary number) inhomogeneity in the rheometer cell is not considered in the comparison to the models, whereas it varies by more than a factor 2 across the gap of the geometry.

Joh et al. [12] have studied both the steady state viscosity and the response to small strain oscillations of bubbly suspensions. Bubbles are said to be formed by "self explosion" in a polyol fluid of viscosity $4 \mathrm{~Pa}$.s, a process leading to a polydisperse suspension with a bubble diameter ranging mostly between 50 and $600 \mu \mathrm{m}$. The volume fraction is varied between 0.1 and 0.3 ; no data is reported in the dilute regime. The suspensions are characterized using a $60 \mathrm{~mm}$ diameter and $2^{\circ}$ angle coneand-plate geometry. This choice looks surprising since the gap between the cone and the plate is only $1 \mathrm{~mm}$ at the edge of the cone with such a geometry, and decreases linearly down to a few tens of microns at the cone truncation. One may thus wonder if the results are really representative of the material bulk behavior; finite size effect such as bubble alignment or even bubble squeezing due to confinement can indeed be expected [13]. They have used the same approach as Llewellin et al. [11] to take into account the suspension polydispersity in the comparison to the models; as mentioned above, there is then an arbitrary choice on the way the contribution of each bubble diameter range to the overall viscosity is estimated, which prevents from drawing final conclusions on the relevance of the existing theoretical models.

Torres et al. [14] have investigated the steady-state and dynamic behaviors of suspensions of bubbles in both Newtonian and nonNewtonian fluids. The Newtonian suspending fluid is honey (viscosity: 5.2 Pa.s), which is aerated with a commercial planetary-action mixer, leading again to a polydisperse suspension, with a bubble diameter ranging between 10 and $270 \mu \mathrm{m}$. The bubbly suspension is studied within a $1 \mathrm{~mm}$ gap parallel-plate geometry; the gap size is thus only 4 times the largest bubble diameter. With a parallel plate geometry, the capillary number varies along the radial dimension, which is not taken into account in the analysis. The studied volume fractions are 13, 14 and 27\%; no data is reported in the dilute regime. The capillary number is varied between $10^{-3}$ and 0.4 ; the high capillary number regime is not studied. The same observations are made as in $[7,11]$. In addition, normal stress differences have been characterized; as in suspensions of hard spheres, they are shown to be proportional to the shear rate at low capillary number.

In order to make significant progress, an original device is designed to produce monodisperse suspensions of bubbles in a highly viscous Newtonian fluid. The shear viscosity of the bubbly suspensions is measured in the dilute and semi-dilute concentration regimes (volume fraction of bubbles between $2 \%$ and $18 \%$ ) using a Couette geometry, for capillary numbers ranging between 0.01 and 10 . The new obtained data are shown to be much more accurate than data previously reported in the literature. Experimental data are compared to the existing models, and are shown to be in very good agreement with the model of Frankel and Acrivos (1970), originally developed for dilute suspensions, in the whole range of studied volume fractions.

\section{Materials and methods}

The materials and methods are designed to: (i) avoid bubble rise during the experiments (this puts constraints on $R / \eta_{0}$, where $R$ is the bubble radius and $\eta_{0}$ is the interstitial fluid viscosity), (ii) obtain data in the range of capillary numbers [0.01 - 10] for typical shear rates 


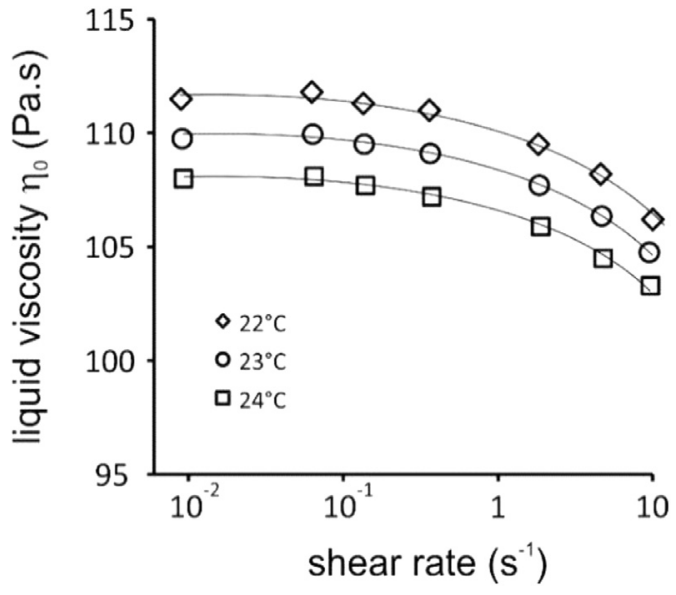

Fig. 1. Shear viscosity as a function of the shear rate for the PDMS oil used as a suspending fluid in the present study for three different temperatures (see legend).

$\dot{\gamma}$ that can be applied (this puts constraints on $\eta_{0} R \dot{\gamma} / \Gamma$ where $\Gamma$ is the air/liquid surface tension), (iii) characterize a representative volume of the material (this puts constraints on $\delta / R$ where $\delta$ is the gap size of the rheometer geometry), (iv) study monodisperse bubbles, (v) obtain volume fraction as high as $20 \%$. With all these constraints in mind, we have studied suspensions of bubbles of radius $350 \mu \mathrm{m}$ in a fluid of viscosity 100 Pa.s, within a Couette geometry of $6 \mathrm{~mm}$ gap, which are prepared in-situ with a home-made setup.

\subsection{Materials}

As a suspending fluid, we use a PDMS oil with a viscosity close to 100 Pa.s (Fungilab RT100000). The oil dynamic viscosity $\eta_{0}$ is measured with a Kinexus Ultra + rheometer (Malvern Instruments) using a thermostated cone-and-plate geometry. Within the investigated range of shear rates, the PDMS oil exhibits a slight shear-thinning behavior, as often found for highly viscous polymeric fluids. The decrease in $\eta_{0}$ values is less than $5 \%$ as the shear rate increases from $0.01 \mathrm{~s}^{-1}$ to $10 \mathrm{~s}^{-1}$, but has to be taken into account as we need to characterize viscosity variations of a few percent when adding bubbles in the dilute regime. Moreover, the effect of temperature is noticeable: $\mathrm{d}\left(\eta_{0}\right) / \mathrm{d} T \approx 2$ Pa.s $/{ }^{\circ} \mathrm{C}$ within the range of temperature $T 22-24^{\circ} \mathrm{C}$ (see Fig. 1 ). In the following, temperature and shear rate dependencies are taken into account when computing the experimental dimensionless viscosity of the bubbly suspension.

It should be noted that the shear-thinning behavior of the oil leads to slight viscosity variations within the gap of the wide-gap Couette geometry used to characterize the bubbly suspensions (see below), since the shear stress varies by a factor close to 2 from the inner to the outer cylinder. These viscosity variations are less than $0.5 \%$. Moreover, since the local shear rate in the interstitial fluid is higher than the macroscopic shear rate, the thinning behavior leads to a slight overestimation of the actual fluid viscosity "seen" by the bubbles. In the range of volume fraction investigated, the local shear rate increase can be estimated [15-17] to be less than $20 \%$, thus leading to a maximum overestimation of the fluid viscosity of $0.2 \%$.

Using a pendant drop tensiometer (Teclis) the surface tension of the PDMS oil is measured to be $\Gamma=20 \pm 1 \mathrm{mN} / \mathrm{m}$.

The gas used to form the bubbles is dinitrogen $\left(\mathrm{N}_{2}\right) .500 \mu \mathrm{m}$ dinitrogen bubbles in PDMS oil are found to be stable with respect to ripening effects over times corresponding to the experiment, i.e. $30 \mathrm{~min}$. Therefore, the bubble size is entirely controlled by the generation process.

\subsection{Generation of the bubbly liquid}

Bubbles are formed in a T-junction with two entries (nitrogen and liquid) and one exit (bubbly liquid). The liquid is pushed at a constant volume flow rate $q_{0}$ using a syringe pump whereas the gas is pushed by applying a constant gas pressure at the entry of the T-junction (typically a differential pressure equal to 2 bars). The $\mathrm{T}$ junction has an internal diameter equal to $1.2 \mathrm{~mm}$, in which a glass capillary of internal diameter $0.5 \mathrm{~mm}$ has been inserted. Outflow tubes have an internal diameter equal to $2 \mathrm{~mm}$ and the apparent shear rate of the fluid is of the order of $1 \mathrm{~s}^{-1}$. Thanks to the flow focusing mechanism [18], small volumes of gas and liquid pass alternatively through the junction. The bubble size is expected to be set by the internal diameter of the T-junction as well as the liquid flow rate and the gas pressure. However, due to the high viscosity of the liquid, the flow configuration is found to be stable only within a limited range of the flow parameters for a given internal diameter, so that we are not able to tune easily the bubble size. In this study we generate steadily monodisperse assemblies of bubbles (see Fig. 2) with a radius close to $350 \mu \mathrm{m}$. We notice a small rate, i.e. $<5 \%$, of coalescence events, occurring mainly in the tubing between the generator and the measurement cell. As explained in the following, the measurement procedure requires that we reproduce several times the same sample, with the same bubble size. The bubble size is measured by image analysis of a monolayer of the foam bubbles squeezed between two glass slides separated by spacers of known thickness. From preliminary measurements for the bubble size produced for several runs with several generators, the bubble radius $R=350 \pm 30 \mu \mathrm{m}$ used for this study corresponds to the observed average value and the observed deviation. Similarly, the measured volume fraction of gas incorporated in the liquid is $\phi_{0}=0.14 \pm 0.01$ at atmospheric pressure. This value results directly from the bubble generation frequency and bubble size, so we are not able to tune it. As a consequence our approach consists in using simultaneously $n$ identical T-junctions, where $n_{b}$ of them are producing bubbles at $\phi_{0}$ and each other junction (numbered $i \in\left[1, n-n_{b}\right]$ ) is injecting liquid at $q_{i}$ without any bubble. In most of experiments, $2<n<4$ and the resulting gas volume fraction is given by $\phi=\phi_{0} /\left(1+\sum_{i} q_{i}\left(1-\phi_{0}\right) / n_{b} q_{0}\right)$. Note that (i) $\phi_{0}$ is the maximum gas volume fraction that can be achieved with our generation setup, and that (ii) in practice, $q_{0} \approx 0.3 \mathrm{~mL} / \mathrm{min}$.

\subsection{Filling of the measurement cell}

Due to the absence of surfactant for stabilizing the bubbles in the PDMS oil, it is necessary to manipulate the bubbly liquid with great care during the filling of the measurement cell, in order to avoid breaking bubbles or inducing coalescence. We have therefore developed a dedicated measurement cell and a filling method which can be described as follows. The measurement cell is a Couette cylinder cell: inner (rotating) cylinder $r_{i}=12.5 \mathrm{~mm}$, outer cylinder $r_{o}=18.5 \mathrm{~mm}$, height $h=40 \mathrm{~mm}$; the walls of the cylinders are serrated to avoid wall slip and bubble alignment along the surfaces. The cell allows the filling from the bottom (see Fig. 2) thanks to a lid which locks the inner cylinder of the cell and also prevents the liquid from flowing out. During the filling step, the cell is mounted on a rotation stage coupled to a vertical translation stage, producing a helical displacement which allows for the bubbly liquid from the generators to be deposited inside the gap of the cell without significant shear stress. When the cell is filled, a bottom is put in place, the cell position is reversed and the cell is set in the rheometer. The inner cylinder is attached, the lid is removed and the measurement can start.

In considering the useful volume to be filled in the measurement cell, i.e. about $30 \mathrm{~mL}$, the filling time varies from $12 \mathrm{~min}\left(q_{0}=0.3 \mathrm{~mL} / \mathrm{min}\right.$, $\sum q_{i}=2.2 \mathrm{~mL} / \mathrm{min}$ and $\left.\phi=0.02\right)$ to $20 \mathrm{~min}\left(4 \times q_{0}=1.2 \mathrm{~mL} / \mathrm{min}\right.$ and $\stackrel{\mathrm{i}}{\phi}=0.15)$. Those filling times are compatible with the slow rising process of the bubbles in the PDMS oil. Indeed, the rising velocity of a single bubble, which provides an upper bound for the bubbly suspension, is 


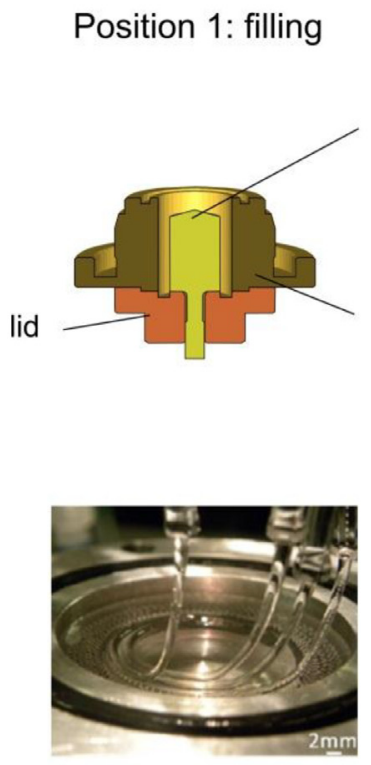

\section{Position 2: measurement}
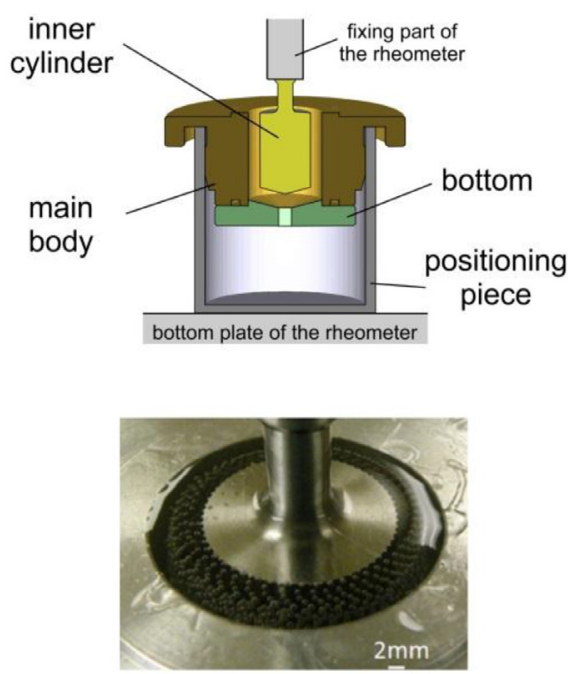

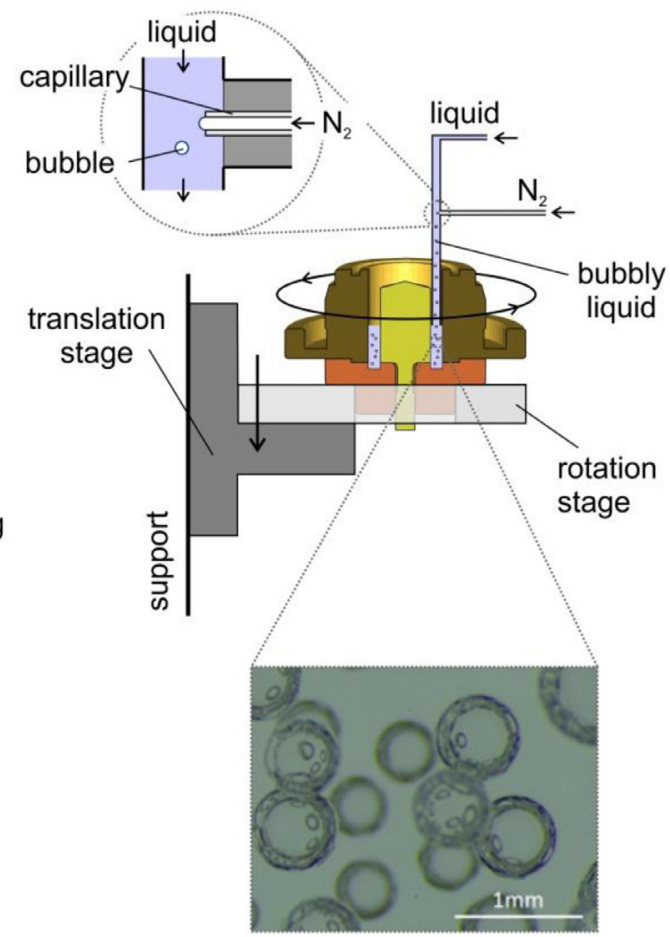

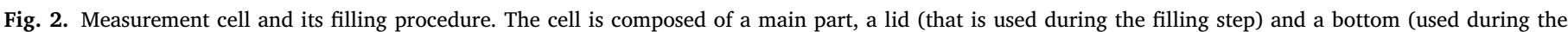

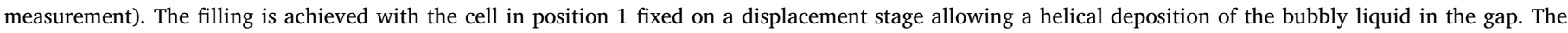
pictures show the filling step ( 4 tubes feed material inside the cell), the measurement step, and the bubbles in the bubbly liquid.

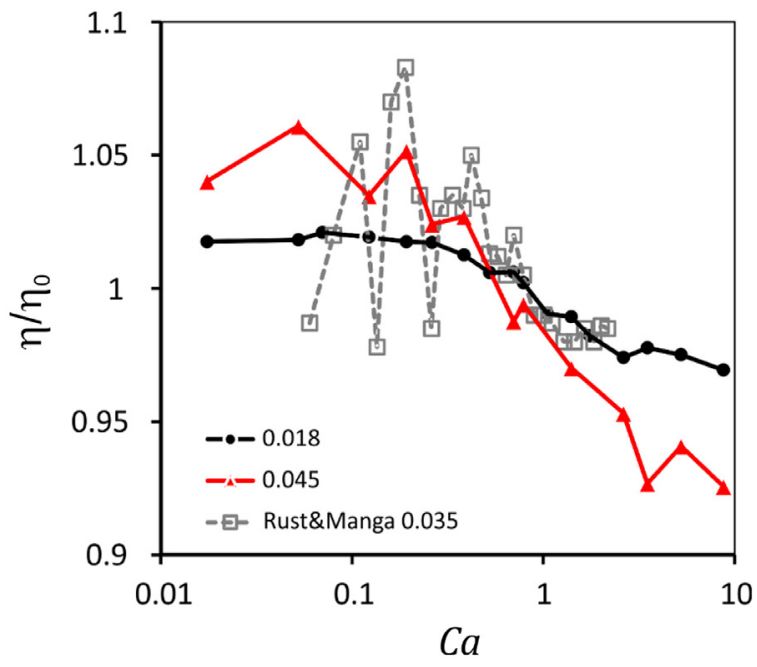

Fig. 3. Dimensionless apparent viscosity $\eta_{s, a p p} / \eta_{0}$ of dilute bubbly suspensions versus capillary number $\mathrm{Ca}$ for two volume fractions $(\phi=0.018 \pm 20 \%, \phi=$ $0.045 \pm 20 \%$, see legend). Previous data from Rust and Manga [7] for $\phi=0.035$ are also plotted.

given by $v_{b}=\pi \Delta \rho g R^{2} / 3 \eta_{0}$ [19], so that within our experimental conditions $v_{b} \approx 10^{2} R^{2} \approx 10 \mu \mathrm{m} / \mathrm{s}$. Thus the average rising height of the first deposited bubble layer is $L \approx 10^{5} R^{2} \sim 10^{-2} \mathrm{~m}$. Note that (i) this height of liquid without bubble is partly contained in the lid of the cell, that is removed afterwards (see Fig. 2), and (ii) due to the helical deposition method, the bubble assembly remains uniform with a modified gas volume fraction that can be estimated to be $\phi(h+L) / h$. Depending on the filling time, the gas volume fraction in the cell is found to vary between $1.1 \phi$ and $1.25 \phi$, which finally allows us to study values of bubble

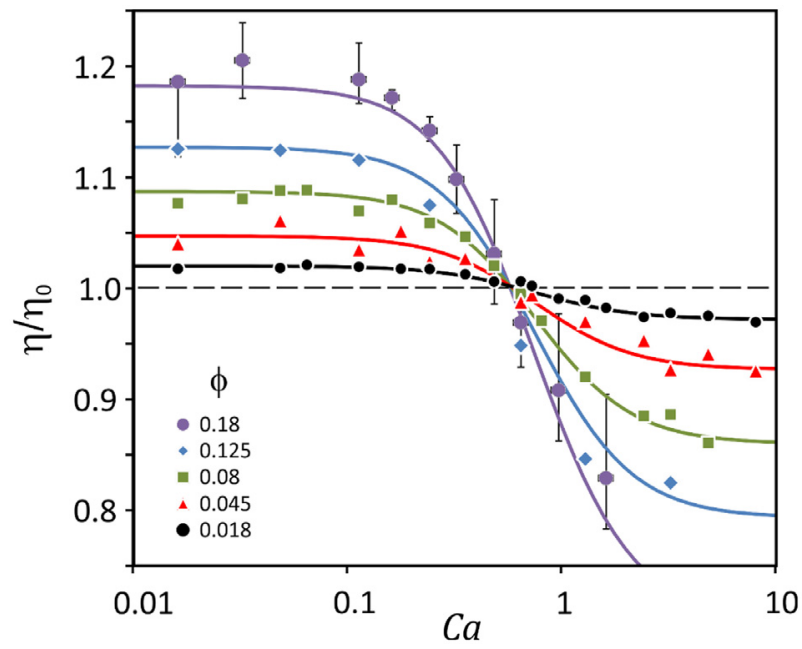

Fig. 4. Dimensionless apparent viscosity $\eta_{s, a p p} / \eta_{0}$ of dilute bubbly suspensions versus capillary number $\mathrm{Ca}$ for all volume fractions investigated (see legend). The predictions of the Frankel and Acrivos (1970) model are also plotted on the same graph, using the following $\phi$ values: 0.018, 0.045, 0.085, 0.125, 0.18.

volume fraction as high as $19 \%$. This effect is taken into account in the following.

\subsection{Rheological measurements}

The viscosity of the bubbly liquid is measured within steady flow conditions at applied torque $T$, and the response of the material obtained through the measurement of the resulting rotation velocity of the inner cylinder $\Omega$. The shear stress $\tau$ at a radial position $r$ in gap of the Couette 

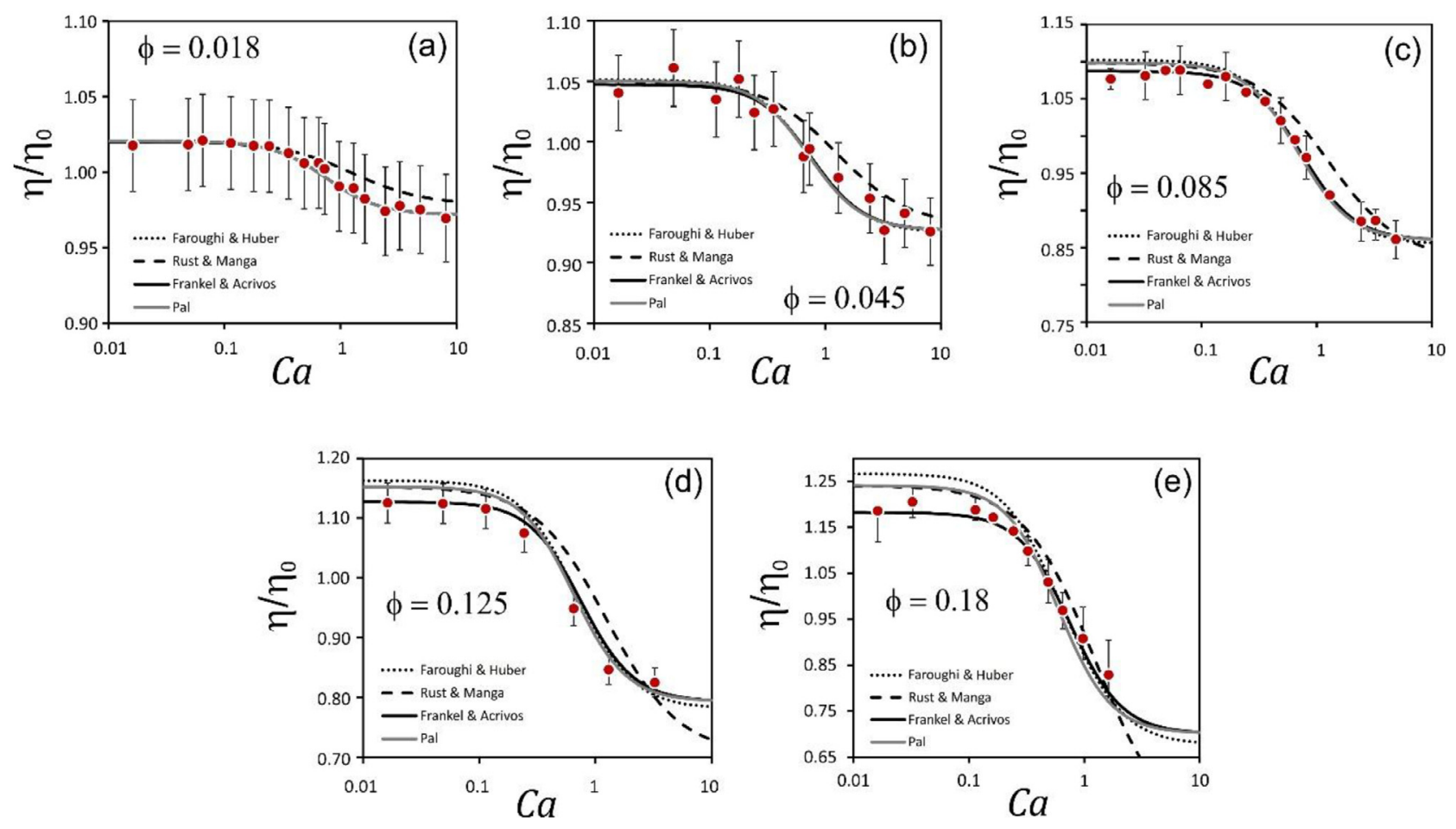

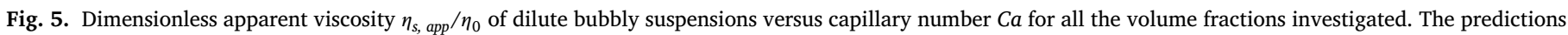

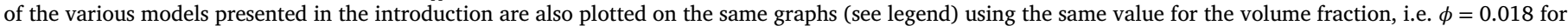
(a), $\phi=0.045$ for (b), $\phi=0.085$ for (c), $\phi=0.125$ for (d), and $\phi=0.18$ for (e).

cell (Fig. 2) is

$\tau(r)=T / 2 \pi h r^{2}$

which implies that the local capillary number is $C a(r)=\tau(r) R / \Gamma=$ $T R / 2 \pi h \Gamma r^{2}$. In the following, when discussing the data, we refer to the values of $\tau(r)$ and $\mathrm{Ca}(r)$ estimated at the average position $\bar{r}=\left(r_{i}+r_{o}\right) / 2$. The shear rate at $\bar{r}$ will be evaluated as its apparent value

$\dot{\gamma}_{a p p}(\bar{r})=\Omega r_{o}^{2} r_{i}^{2} /\left[\overline{\mathrm{r}}^{3}\left(r_{o}-r_{i}\right)\right]$

which is exact only for a Newtonian behavior. We will then report apparent viscosity data $\eta_{s, a p p}(\overline{C a})=\tau(\bar{r}) / \dot{\gamma}_{a p p}(\bar{r})$.

The spatial variations of $\mathrm{Ca}$ within the gap are of order $80 \%$. For a quantitative comparison of the experimental data and the models, these spatial variations need to be accounted for. For each of the models, the behavior of the theoretical bubbly suspension in the studied wide gap Couette cell will thus be computed to compute the theoretically expected $\eta_{s, a p p}^{\text {th }}(\overline{C a})$, which will finally be compared to the experimental $\eta_{s, a p p}(\overline{C a})$; details on these computations are provided in the next subsection.

In contrast to the experiment performed by Rust and Manga [7], it was not possible to measure continuously, i.e. with the same sample, the viscosity corresponding to increasing values of $\mathrm{Ca}$. Indeed, after several rotations of the inner cylinder we observed large gas bubbles rising from the bulk sample and bursting at the free surface. This phenomenon is due to the fact that the bubbles interface is not stabilized against coalescence, and this effect is all the more pronounced that the $\mathrm{Ca}$ value is high and that the gas volume fraction is high. Therefore, we start the measurement for a low value of $\mathrm{Ca}$ (typically 0.1 ) by applying a constant stress, then we measure the viscosity for one or several larger $\mathrm{Ca}$ values by applying various constant stress values and we return to the first low $C a$ value in order to check that the viscosity did not change, i.e. that the sample remained homogenous during the measurement at higher $\mathrm{Ca}$ value. For each measurement, a constant stress is applied during $30 \mathrm{~s}$, which was sufficient in all cases to reach a steady state.

We notice that for the largest investigated gas volume fractions, it was not possible to measure the viscosity corresponding to $C a \gtrsim 1$ with- out observing large bubbles rising; this points to the impact of bubble shape on the rise velocity of a bubble assembly. In the following we report results for those where large bubble rising was not observed. Five small ranges of gas volume fractions have been considered: $\phi=0.018 \pm 20 \%, \phi=0.045 \pm 20 \%, \phi=0.085 \pm 15 \%, \phi=0.125 \pm 12 \%$, and $\phi=0.18 \pm 10 \%$. For each range of volume fraction, 5 samples were prepared; in the following, at each $\phi$, we show the data averaged over the 5 samples.

\subsection{Model analysis}

As stated above, in order to make a quantitative comparison possible between the experimental measurements and the models, we need to account for the stress inhomogeneity in the Couette device. Indeed, the model predictions are for homogeneous simple shear flows, whereas experimental data are apparent viscosity data $\eta_{s, a p p}(\overline{C a})=\tau(\bar{r}) / \dot{\gamma}_{a p p}(\bar{r})$ in an inhomogeneous stress field. In the following, we thus show how a prediction $\eta_{s, a p p}^{t h}(\overline{C a}, \phi)$ can be computed from the theoretical prediction $\eta_{s}(\mathrm{Ca}, \phi)$ for the viscosity of a bubbly suspension of given volume fraction $\phi$.

We first note that, for a given torque $T$ applied to the rheometer, $\tau(r)=T / 2 \pi h r^{2}$, which does not depend on the material. The actual values of $\dot{\gamma}(r)$ and of the resulting macroscopic measurement $\Omega=$ $\dot{\gamma}_{a p p}(\vec{r})\left[\vec{r}^{3}\left(r_{o}-r_{i}\right)\right] /\left[r_{o}^{2} r_{i}^{2}\right]$, however, are not known a priori and need to be computed for each constitutive behavior. In order to compute $\eta_{s, a p p}^{t h}(\overline{C a}, \phi)$, we need to know the value of $\dot{\gamma}_{a p p}^{\text {th }}(\bar{r})$ for a given $\eta_{s}(C a$, $\phi)$. We first recall that $\dot{\gamma}(r)=-r \partial_{r}(\omega(r))$ where $\omega(r)$ is the local rotation velocity. We then write $\int_{r_{i}}^{r_{0}} \dot{\gamma}(r) / r \mathrm{~d} r=\int_{r_{i}}^{r_{0}}-\partial_{r}(\omega(r)) \mathrm{d} r=\Omega$ by using the boundary conditions $\omega\left(r_{o}\right)=0$ at the outer cylinder and $\omega\left(r_{i}\right)=\Omega$ at the inner cylinder. Replacing $\Omega$ by $\dot{\gamma}_{a p p}^{t h}(\bar{r})\left[\bar{r}^{3}\left(r_{o}-r_{i}\right)\right] /\left[r_{o}^{2} r_{i}^{2}\right]$ and $\dot{\gamma}(r)$ by its actual value $\tau(r) / \eta_{s}(C a, \phi)$, we then obtain: $\int_{r_{\mathrm{i}}}^{r_{0}} \tau(r) /\left[r \eta_{s}(C a, \phi)\right] \mathrm{d} r=$ $\dot{\gamma}_{a p p}(\bar{r})\left[\bar{r}^{3}\left(r_{o}-r_{i}\right)\right] /\left[r_{o}^{2} r_{i}^{2}\right]$. Noting that $\tau(r)=\tau(\bar{r}) \times \bar{r}^{2} / r^{2}$ and $C a(r)=$ 
$\overline{C a} \times \bar{r}^{2} / r^{2}$, we end up with the following equation for $\eta_{s, a p p}^{t h}(\overline{C a}, \phi)=$ $\tau(\bar{r}) / \dot{\gamma}_{a p p}^{\text {th }}(\bar{r}):$

$\eta_{s, a p p}^{\text {th }}(\overline{C a}, \phi)=\left[\bar{r}\left(r_{o}-r_{i}\right) /\left(r_{o}^{2} r_{i}^{2}\right)\right] / \int_{r_{i}}^{r_{0}} 1 /\left[r^{3} \eta_{s}\left(\overline{C a} \times \frac{\bar{r}^{2}}{r^{2}}, \phi\right)\right] \mathrm{d} r$

which needs to be computed for each theoretical behavior $\eta_{s}(\mathrm{Ca}, \phi)$. As an example, the Frankel and Acrivos (1970) equation yields the following development at first order in $\phi$ for $\eta_{s, a p p}^{\text {th }}(\overline{C a}, \phi)$ :

$\eta_{s, a p p}^{\text {th }}(\overline{C a}, \phi)=1-\frac{5 \phi}{3}+\frac{20 \phi}{9} \times \frac{\hat{r}_{0}^{2} \hat{r}_{i}^{2}}{\hat{r}_{0}^{2}-\hat{r}_{i}^{2}} \times \frac{1}{\overline{C a}} \times \arctan \left(\frac{30\left(\hat{r}_{0}^{2}-\hat{r}_{i}^{2}\right) \overline{C a}}{25 \hat{r}_{0}^{2} \hat{r}_{i}^{2}+36 \overline{C a}^{2}}\right)$

where $\hat{r}=r / \bar{r}$.

\section{Results and discussion}

In Fig. 3 we plot the apparent viscosity data obtained in the dilute regime $(\phi<5 \%)$ together with previous data from Rust and Manga [7].

It is first observed that we successfully managed to obtain data much more accurate than previously reported and for a wider range of capillary numbers. This opens the possibility to test accurately the proposed models, in particular in the dilute limit. We now switch to the presentation of all of our data together with their comparison to the models presented in the introduction.

In Fig. 4, we observe all the features of bubbly suspensions reported in the literature: (i) the dimensionless viscosity $\eta_{s, a p p} / \eta_{0}$ is a decreasing function of the capillary number $\mathrm{Ca}$, with a plateau at low $\mathrm{Ca}$ that is readily observable for $\mathrm{Ca}<0.1$, whereas the plateau at large $\mathrm{Ca}$ is not observed and should thus be attained for $C a>10$, (ii) $\eta_{s, \text { app }} / \eta_{0}$ is an increasing function of $\phi$ at low $\mathrm{Ca}$ and a decreasing function of $\phi$ at high $\mathrm{Ca}$, and (iii) there is a crossover of all curves at a same point $\left(\mathrm{C} a_{c} \simeq\right.$ $\left.0.6, \eta_{s, a p p} / \eta_{0}=1\right)$ consistent with the theoretical prediction for the dilute regime (Eq. (9)).

It is also remarkable here that the Frankel and Acrivos (1970) model provides a good agreement with all of our data, whereas it is a development at first order in $\phi$ only, which is not $a$ priori expected to be valid for volume fractions larger than $\simeq 5 \%$. The same feature was observed for the elastic modulus of soft aerated solids [16].

In order to test more accurately all of the models, we now plot separately in Fig. 5 the dimensionless viscosity $\eta_{s, ~ a p p} / \eta_{0}$ data for all investigated volume fractions and compare each of the curves to Eqs. (6)-(8) and (10).

In the dilute regime $(\phi=0.018)$, all the theoretical models, which are consistent with the Frankel and Acrivos (1970) model at first order in $\phi$ are observed to be in good agreement with the data. However, the empirical model of Rust and Manga [7] is already seen here to tend to overestimate the value of the viscosity of the bubbly suspension for deformable bubbles $(\mathrm{Ca}=\mathrm{O}(1))$.

As the concentration is increased, the tendency of the Rust and Manga (2002) model to overestimate the viscosity of deformable bubbles is confirmed. At the highest volume fraction investigated, it also overestimates the viscosity of undeformed bubble $(\mathrm{Ca}<0.1)$. This also seems to be the case of the Faroughi and Huber (2015) and the Pal (2004) model, although it is hard to conclude given the uncertainty of the measurements.

The Frankel and Acrivos (1970) model finally seems to provide the best agreement with the data in the whole range of volume fraction and capillary numbers investigated.

\section{Conclusion}

In order to make significant progress as compared to results available in the literature, an original device was designed to produce monodisperse suspensions of bubbles in a highly viscous Newtonian fluid. The viscosity of the bubbly suspensions was measured in the dilute and semidilute concentration regimes (volume fraction of bubbles between $2 \%$ and $18 \%$ ) using a Couette geometry, for capillary numbers ranging between 0.01 and 10 . The new obtained data were shown to be much more accurate than data previously reported in the literature, thus allowing for a comparison with theoretical models. Classical features of bubbly suspensions have been observed: (i) the dimensionless viscosity $\eta_{s} / \eta_{0}$ is a decreasing function of the capillary number $\mathrm{Ca}$, with a plateau at low $\mathrm{Ca}$ that is readily observable for $\mathrm{Ca}<0.1$, whereas the plateau at large $\mathrm{Ca}$ is not observed and should thus be attained for $\mathrm{Ca}>10$, (ii) $\eta_{s} / \eta_{0}$ is an increasing function of $\phi$ at low $\mathrm{Ca}$ and a decreasing function of $\phi$ at high $\mathrm{Ca}$, and (iii) there is a crossover of all curves at a same point ( $C a=0.6, \eta_{s} / \eta_{0}=1$ ) consistent with the theoretical prediction for the dilute regime. Over the whole range of capillary numbers and volume fraction investigated, the best agreement was found with the model of Frankel and Acrivos [4], originally designed for dilute suspensions, and which was thus not expected to be valid at volume fraction as high as $20 \%$.

\section{References}

[1] M.M. Denn, J.F. Morris, Rheology of non-Brownian suspensions, Annu. Rev. Chem. Biomol. Eng. 5 (2014) 203-228.

[2] A. Einstein, Eine neue Bestimmung der Molekuldimensionen, An. Phys. 19 (1906) 289-306 English translation in Investigation on the Theory of Brownian Motion, Dover, New York, 1956.

[3] G.I. Taylor, The viscosity of a fluid containing small drops of another fluid, Proc. R. Soc. London Ser. A. 138 (1932) 41-48.

[4] N.A. Frankel, A. Acrivos, The constitutive equation for a dilute emulsion, J. Fluid Mech. 44 (1970) 65-78.

[5] J.G. Oldroyd, The elastic and viscous properties of emulsions and suspensions, Proc R. Soc. London Ser. A. 218 (1953) 122-132.

[6] S.J. Choi, W.R. Schowalter, Rheological properties of nondilute suspensions of deformable particles, Phys. Fluids 18 (1975) 420-427.

[7] A.C. Rust, A. Manga, Effects of bubble deformation on the viscosity of dilute suspensions, J. Non-Newton. Fluid Mech. 104 (2002) 53-63.

[8] R. Pal, Rheological constitutive equation for bubbly suspensions, Ind. Eng. Chem. Res. 43 (2004) 5372-5379.

[9] S.A. Faroughi, C. Huber, A generalized equation for rheology of emulsions and suspensions of deformable particles subjected to simple shear at low Reynolds number, Rheol. Acta 54 (2015) 85-108.

[10] R. Lespiat, S. Cohen-Addad, R. Höhler, Jamming and flow of random-close-packed spherical bubbles: an analogy with granular materials, Phys. Rev. Lett. 106 (2011) 148302.

[11] E. Llewellin, H. Mader, S. Wilson, The rheology of a bubbly liquid, Proc. R. Soc. London Ser. A. 458 (2002) 987-1016.

[12] S.W. Joh, S.H. Lee, J.R. Youn, Rheological behavior of polydispersed bubble suspensions in shear flows, Polym. Eng. Sci. 50 (2010) 128-137.

[13] R. Morini, X. Chateau, G. Ovarlez, O. Pitois, L. Tocquer, Viscous friction of squeezed bubbly liquid layers, Soft Matter 14 (2018) 8372-8377.

[14] M.D. Torres, F. Gadala-Maria, D.I. Wilson, Comparison of the rheology of bubbly liquids prepared by whisking air into a viscous liquid (honey) and a shear-thinning liquid (guar gum solutions), J. Food Eng. 118 (2013) 213-228.

[15] X. Chateau, G. Ovarlez, K.L. Trung, Homogenization approach to the behavior of suspensions of noncolloidal particles in yield stress fluids, J. Rheol. 52 (2008) 489-506.

[16] L. Ducloué, O. Pitois, J. Goyon, X. Chateau, G. Ovarlez, Coupling of elasticity to capillarity in soft aerated materials, Soft Matter 10 (2014) 5093-5098.

[17] L. Ducloué, O. Pitois, J. Goyon, X. Chateau, G. Ovarlez, Rheological behaviour of suspensions of bubbles in yield stress fluids, J. Non-Newton. Fluid Mech 215 (2015) 31-39.

[18] A.M. Gañán-Calvo, J. Gordillo, Perfectly monodisperse microbubbling by capillary flow focusing, Phys. Rev. Lett. 87 (2001) 274501.

[19] J.S. Hadamard, Mouvement permanent lent d'une sphère liquide et visqueuse dans un liquide visqueux, C. R. Acad. Sci. 152 (1911) 1735-1738. 